\title{
Supporting Information: Simulations of Ice Nucleation by Model AgI Disks and Plates
}

Stephen A. Zielke, Allan K. Bertram, and G. N. Patey

Department of Chemistry, University of British

Columbia, Vancouver, BC, Canada V6T $1 Z 1$

(Dated: January 16, 2016) 
Estimates of the bulk water densities at $300 \mathrm{~K}$ in the systems considered are given in Table S1. These are obtained from water density profiles far from the AgI surface, where surface effects are no longer detectable. The simulations used in this paper are listed in Table S2 for the six-site model, and in Table S3 for the TIP4P/2005 model. For each simulation, the system, temperature, and length of the simulation are given. Whether or not ice nucleated is indicated in the last column.

Plots of ice cluster size versus time for system C2 are shown in Figure S1. Note that the C2 system has two AgI disks, so each simulation has two ice clusters that eventually join after ice nucleates, and grows to fill the simulation cell.

In our simulations, we might expect the AgI disks and plates to give rise to an electric field. Large electric fields that highly polarize water can induce ice nucleation ${ }^{1}$. Therefore, to check this possibility we calculated polarization profiles ${ }^{1}$

$$
\langle\boldsymbol{m}(Z)\rangle=\left\langle\frac{1}{N(Z) \mu_{w}} \sum_{i=1}^{N(Z)} \boldsymbol{\mu}_{i}\right\rangle
$$

where $\langle\boldsymbol{m}(Z)\rangle$ is the net polarization (mean reduced dipole moment) in a rectangular volume element of width $0.01 \mathrm{~nm}$ at $Z, N(Z)$ is the number of water molecules in the volume element, $\mu_{w}$ is the molecular dipole moment of the water model, and $\boldsymbol{\mu}_{i}$ is the dipole vector of molecule $i$. The components of $\langle\boldsymbol{m}(Z)\rangle$ are plotted in Figure S6.

The plots show that the net polarization of water is too small to significantly influence ice nucleation. This is confirmed by the fact that systems $\mathrm{C}$ and $\mathrm{C} 2$ (in $\mathrm{C} 2$ the fields from the disks cancel, such that there is no net polarization away from the disk surfaces) give very similar nucleation results.

\section{A. Comparison of force fields}

In addition to the Lennard-Jones and Coulombic terms used in our present and previous ${ }^{2}$ simulations, the original force field proposed by Hale and Kiefer $^{3}$ for a water molecule interacting with an AgI surface included a polarization interaction of the general form

$V_{\mathrm{IND}}=-\frac{1}{8 \pi \epsilon_{o}} \sum_{n} \sum_{m} \alpha_{w} \frac{Q_{n} Q_{m}\left(\boldsymbol{r}_{o}-\boldsymbol{r}_{n}\right)\left(\boldsymbol{r}_{o}-\boldsymbol{r}_{m}\right)}{\left|\boldsymbol{r}_{o}-\boldsymbol{r}_{n}\right|^{3}\left|\boldsymbol{r}_{o}-\boldsymbol{r}_{m}\right|^{3}}-\frac{1}{8 \pi \epsilon_{o}} \sum_{m} \sum_{i} \sum_{j} \alpha_{m} \frac{Q_{i} Q_{j}\left(\boldsymbol{r}_{o}-\boldsymbol{r}_{i}\right)\left(\boldsymbol{r}_{o}-\boldsymbol{r}_{j}\right)}{\left|\boldsymbol{r}_{o}-\boldsymbol{r}_{m}\right|^{3}\left|\boldsymbol{r}_{o}-\boldsymbol{r}_{m}\right|^{3}}$, 
where $\epsilon_{o}$ is the permittivity of free space, $\alpha_{w}$ and $\alpha_{m}$ are the polarizabilities of water and of Ag or I ions, respectively, $Q_{k}$ represents the partial charge at a site $k$ on the AgI lattice or on the water molecule, $\boldsymbol{r}_{k}$ is the position vector of any site $k$, and $\boldsymbol{r}_{o}$ is the position vector of the oxygen atom of the water molecule. The sums on $n$ and $m$ are over all $\mathrm{Ag}$ and I ions in the AgI lattice, and the sums on $i$ and $j$ are over all charge sites in the water model. The first term in Eq. (2) represents the interaction of the AgI lattice with the induced dipole moment of the water molecule, and the second term is the interaction of the water molecule with the dipole moment induced in a Ag or I ion.

In their calculations Hale and Kiefer ${ }^{3}$ actually used an approximate form of the AgIwater induction potential, retaining only the $m=n$ terms in the first term of Eq. (2). They note that this is a good approximation because the $m \neq n$ terms contribute only about $\sim 5 \%$ to the total potential. This simplification means that the water-molecule-AgI-surface interaction can be written as a sum of pairwise interactions

$$
V_{\mathrm{IND}}=\sum_{m} \tilde{V}_{\mathrm{IND}}
$$

where the sum on $m$ is over all Ag and I ions, and $\tilde{V}_{\text {IND }}$ depends on the water-ion distance and on the orientation of the water molecule. Note that the induction potential is still not pairwise additive with respect to the multiple charge sites in the water model [note the sums on $i$ and $j$ in the second term of Eq. (2)], which means that $\tilde{V}_{\text {IND }}$ cannot be efficiently included in parallel MD algorithms, however, it does allow us to meaningfully compare total Ag-water and I-water pair potentials both with and without induction effects.

Results obtained for the six-site water model for three orientations of the water molecule (see Fig. S8) are shown in Fig. S9. We note that results for the TIP4P/ice model (not shown) are very similar. In Fig. S9 four curves are shown for each orientation of the water molecule. The Hale-Kiefer $(\mathrm{HK})$ potentials with $\mathrm{Ag}$ and I partial charges of $\pm 0.6 \mathrm{e}$ and including $\tilde{V}_{\text {IND }}$ are represented by green curves. HK potentials neglecting $\tilde{V}_{\text {IND }}$, as used in the present paper, are shown as black curves. Additionally, we include curves for potentials that neglect $\tilde{V}_{\text {IND }}$ but have Ag and I partial charges of zero (blue curves) and $\pm 0.8 \mathrm{e}$ (red curves). In an earlier paper ${ }^{2}$ we showed that AgI slabs modelled with partial charges ranging from zero to \pm 0.8 e all nucleated ice in a similar manner. From Fig. S9, we see that while the HK potential does have a significant contribution from $\tilde{V}_{\text {IND }}$ (particularly for attractive 
orientations) it does not fall outside the range of potentials for which we have observed ice nucleation. In particular, for the important orientations of the water molecule where the interactions are strongly attractive, the HK potential is comparable with the potential obtained with partial charges of $\pm 0.8 \mathrm{e}$. Therefore, based on our observation of ice nucleation for a wide range of Coulombic interactions, including the three cases shown in Fig. S9, we do not believe that using the full HK potential (if that were computationally feasible) would qualitatively alter our results or conclusions.

1 Yan, J. Y.; Patey, G. N. Molecular Dynamics Simulations of Ice Nucleation by Electric Fields. J. Phys. Chem. A 2012. 116, 7057-7064.

2 Zielke, S. A.; Bertram, A. K.; Patey, G. N. A Molecular Mechanism of Ice Nucleation on Model AgI Surfaces. J. Phys. Chem. B 2015. DOI: 10.1021/jp508601s.

3 Hale, B. N.; Kiefer, J. Studies of $\mathrm{H}_{2} \mathrm{O}$ on $\beta$-AgI Surfaces: An Effective Pair Potential Model. $J$. Chem. Phys. 1980. 73, 923-933. 
TABLE S1. Bulk water densities of the systems (six-site model) considered at $300 \mathrm{~K}$, measured far from the disk surface.

\begin{tabular}{cc}
\hline System & Density $\left(\mathrm{kg} / \mathrm{m}^{3}\right)$ \\
\hline A & 947 \\
B & 947 \\
C & 952 \\
C2 & 947 \\
D & 945 \\
Dr & 945 \\
E & 953 \\
\hline
\end{tabular}


TABLE S2. A list of simulations performed with the six-site model. Systems marked with a 'P, were conducted at 1 bar pressure and a ${ }^{\circ}{ }^{\circ}$ indicates the $\mathrm{Ag}$ and I atoms were neutral.

\begin{tabular}{|c|c|c|c|c|c|c|c|}
\hline System & $\Delta T_{s}(\mathrm{~K})$ & Time (ns) & Nucleated ice? & System & $\Delta T_{s}(\mathrm{~K})$ & Time (ns) & Nucleated ice? \\
\hline $\mathrm{A}$ & 31 & 50 & No & $\mathrm{C} 2$ & 20 & 98 & No \\
\hline $\mathrm{A}$ & 29 & 50 & No & $\mathrm{C} 2$ & 22 & 100 & Yes \\
\hline $\mathrm{A}$ & 34 & 150 & No & $\mathrm{C} 2$ & 21 & 100 & Yes \\
\hline $\mathrm{A}$ & 39 & 102 & Yes & $\mathrm{C} 2$ & 20 & 100 & No \\
\hline $\mathrm{A}$ & 36 & 127 & Yes & $\mathrm{C} 2$ & 19 & 100 & No \\
\hline $\mathrm{A}$ & 37 & 100 & Yes & $\mathrm{C} 2$ & 21 & 100 & Yes \\
\hline $\mathrm{A}$ & 35 & 150 & Yes & $\mathrm{D}$ & 14 & 98 & No \\
\hline $\mathrm{A}$ & 35 & 150 & Yes & $\mathrm{D}$ & 16 & 148 & Yes \\
\hline $\mathrm{A}$ & 34 & 150 & No & $\mathrm{D}$ & 15 & 150 & No \\
\hline $\mathrm{A}^{P}$ & 35 & 300 & Yes & $\mathrm{D}$ & 15 & 150 & No \\
\hline $\mathrm{B}$ & 29 & 98 & Yes & $\mathrm{D}$ & 16 & 123 & Yes \\
\hline B & 24 & 98 & No & $\mathrm{D}$ & 24 & 50 & Yes \\
\hline $\mathrm{B}$ & 26 & 150 & Yes & $\mathrm{D}$ & 29 & 50 & Yes \\
\hline B & 27 & 98 & Yes & $\mathrm{D}$ & 24 & 50 & Yes \\
\hline $\mathrm{B}$ & 25 & 39 & No & $\mathrm{D}$ & 29 & 50 & Yes \\
\hline B & 26 & 248 & Yes & $\mathrm{D}$ & 13 & 100 & No \\
\hline B & 25 & 39 & No & $\mathrm{D}$ & 9 & 100 & No \\
\hline B & 21 & 150 & No & $\mathrm{D}^{P}$ & 18 & 75 & Yes \\
\hline $\mathrm{B}^{P}$ & 26 & 175 & Yes & $\mathrm{D}^{0}$ & 16 & 150 & Yes \\
\hline $\mathrm{C}$ & 19 & 100 & No & $\mathrm{D}^{0}$ & 18 & 75 & Yes \\
\hline $\mathrm{C}$ & 24 & 48 & Yes & Dr & 16 & 115 & Yes \\
\hline $\mathrm{C}$ & 21 & 98 & No & $\mathrm{Dr}$ & 15 & 150 & No \\
\hline $\mathrm{C}$ & 22 & 78 & Yes & $\mathrm{Dr}$ & 16 & 113 & Yes \\
\hline $\mathrm{C}$ & 22 & 78 & Yes & Dr & 16 & 183 & Yes \\
\hline $\mathrm{C}$ & 21 & 98 & No & Dr & 15 & 150 & No \\
\hline $\mathrm{C}$ & 22 & 100 & Yes & $\mathrm{E}$ & 13 & 150 & No \\
\hline $\mathrm{C}$ & 17 & 100 & No & $\mathrm{E}$ & 12 & 150 & No \\
\hline $\mathrm{C}$ & 14 & 100 & No & $\mathrm{E}$ & 14 & 175 & Yes \\
\hline $\mathrm{C}^{P}$ & 22 & 125 & Yes & $\mathrm{E}$ & 15 & 100 & Yes \\
\hline $\mathrm{C}^{0}$ & 22 & 100 & Yes & $\mathrm{E}$ & 9 & 150 & No \\
\hline $\mathrm{C}^{0}$ & 21 & 100 & Yes & $\mathrm{E}$ & 14 & 150 & Yes \\
\hline $\mathrm{C} 2$ & 29 & 48 & Yes & $\mathrm{E}$ & 13 & 150 & No \\
\hline $\mathrm{C} 2$ & 29 & 48 & Yes & & & & \\
\hline $\mathrm{C} 2$ & 24 & 48 & Yes & & & & \\
\hline $\mathrm{C} 2$ & 22 & 48 & Yes & & & & \\
\hline $\mathrm{C} 2$ & 19 & 48 & No & & & & \\
\hline
\end{tabular}


TABLE S3. A list of simulations performed with the TIP4P/2005 model

\begin{tabular}{cccc|cccc}
\hline \multicolumn{2}{c}{ System } & $\Delta T_{s}(\mathrm{~K})$ & Time $(\mathrm{ns})$ & Nucleated ice? & \multicolumn{4}{|c}{ System } & $\Delta T_{s}(\mathrm{~K})$ & Time (ns) & Nucleated ice? \\
\hline $\mathrm{D}$ & 22.5 & 300 & Yes & $\mathrm{E}$ & 20.5 & 400 & Yes \\
$\mathrm{D}$ & 28.5 & 300 & Yes & $\mathrm{E}$ & 15.5 & 300 & No \\
$\mathrm{D}$ & 25.5 & 300 & Yes & $\mathrm{E}$ & 25.5 & 300 & Yes \\
$\mathrm{D}$ & 30.5 & 200 & Yes & $\mathrm{E}$ & 17.5 & 600 & No \\
$\mathrm{D}$ & 20.5 & 600 & Yes & $\mathrm{E}$ & 18.5 & 600 & Yes \\
$\mathrm{D}$ & 21.5 & 500 & Yes & $\mathrm{E}$ & 19.5 & 100 & Yes \\
$\mathrm{D}$ & 19.5 & 500 & Yes & $\mathrm{E}$ & 17.5 & 600 & Yes \\
$\mathrm{D}$ & 18.5 & 500 & No & $\mathrm{E}$ & 18.5 & 500 & Yes \\
$\mathrm{D}$ & 18.5 & 600 & Yes & & & & \\
$\mathrm{D}$ & 19.5 & 500 & No & & & &
\end{tabular}




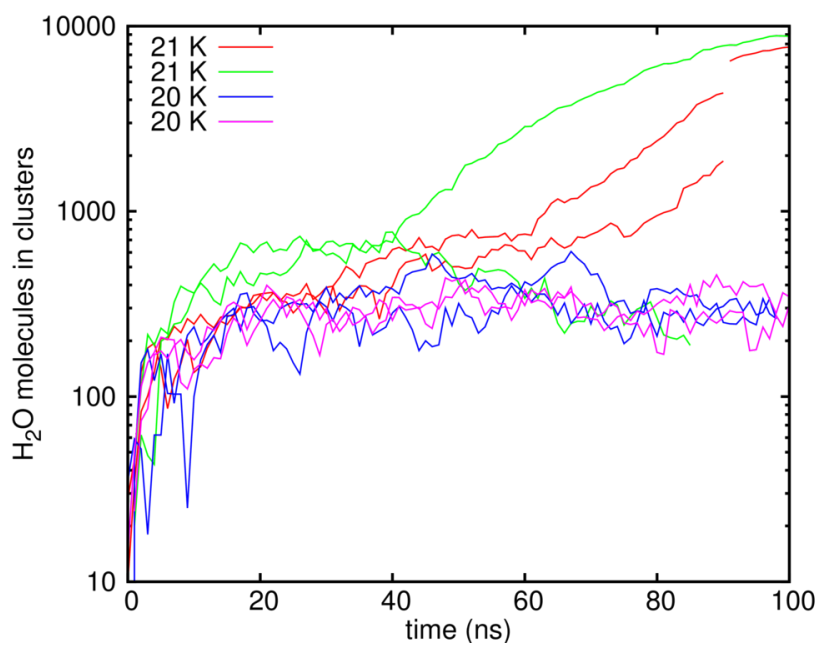

FIG. S1. Ice cluster size versus time for the C2 case (six-site model) at the $\Delta T_{s}$ values shown in the legend. 

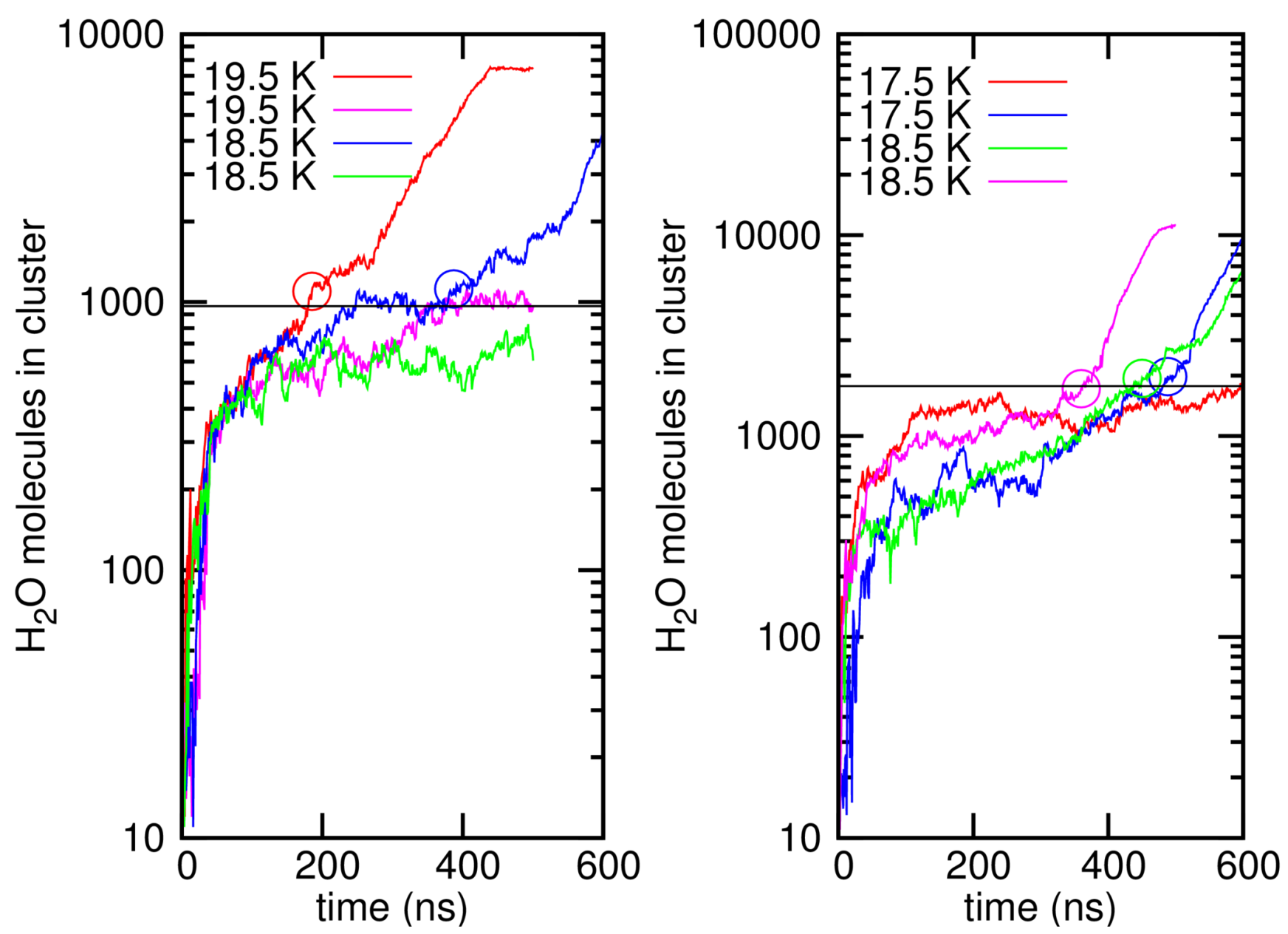

FIG. S2. Ice cluster size versus time for the TIP4P/2005 model. The left panel is for disk D and the right panel for disk E. The horizontal lines indicate our lower limit estimates of the critical cluster size. Estimates of the upper size limit are shown as circles. $\Delta T_{s}$ values are given in the legend. 

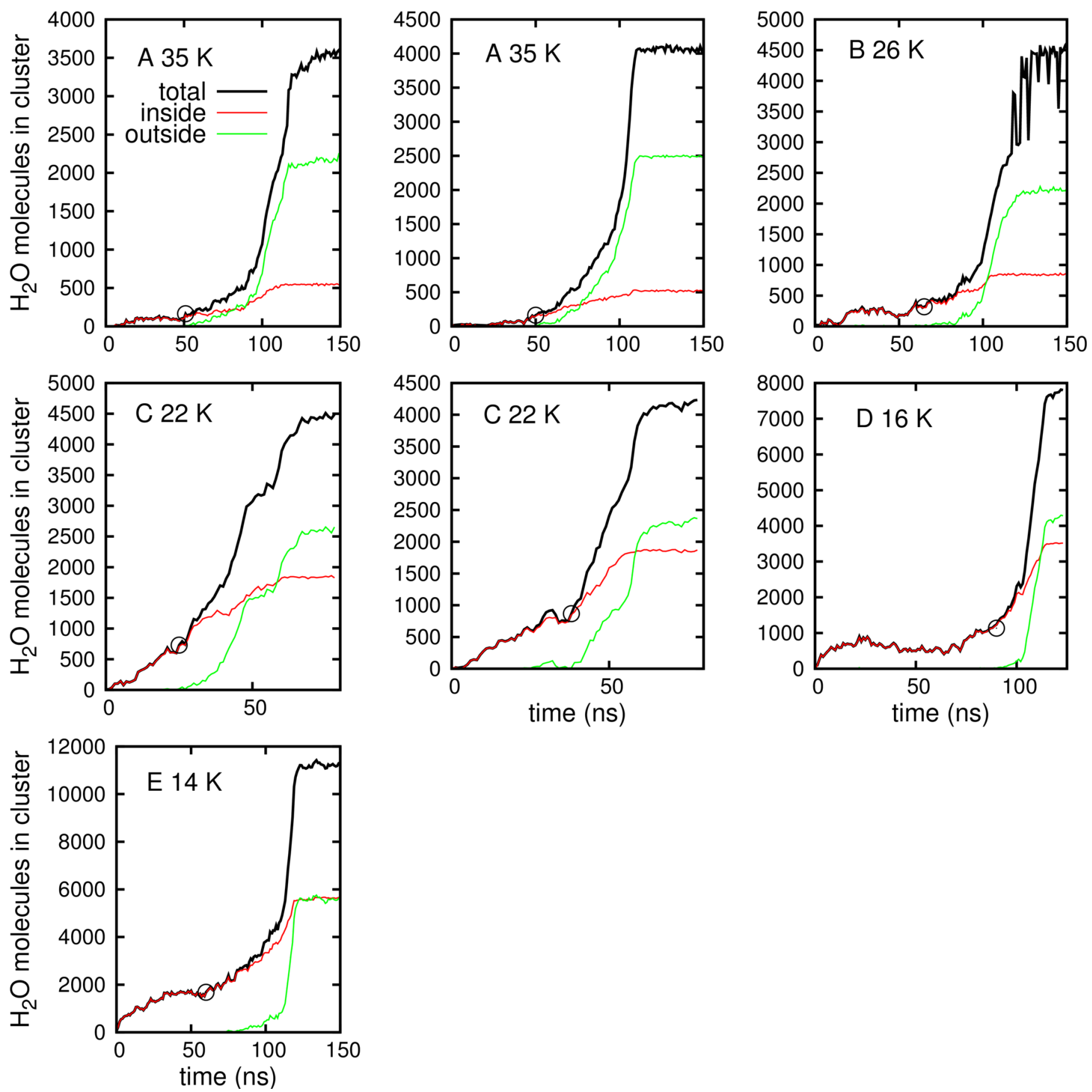

FIG. S3. Ice cluster size versus time for the six-site model. The total cluster size is represented by a black curve, the amounts of ice inside and outside a cylindrical volume defined by the disk radius are red and green, respectively. The system label and the $\Delta T_{s}$ value are given in the legend. 

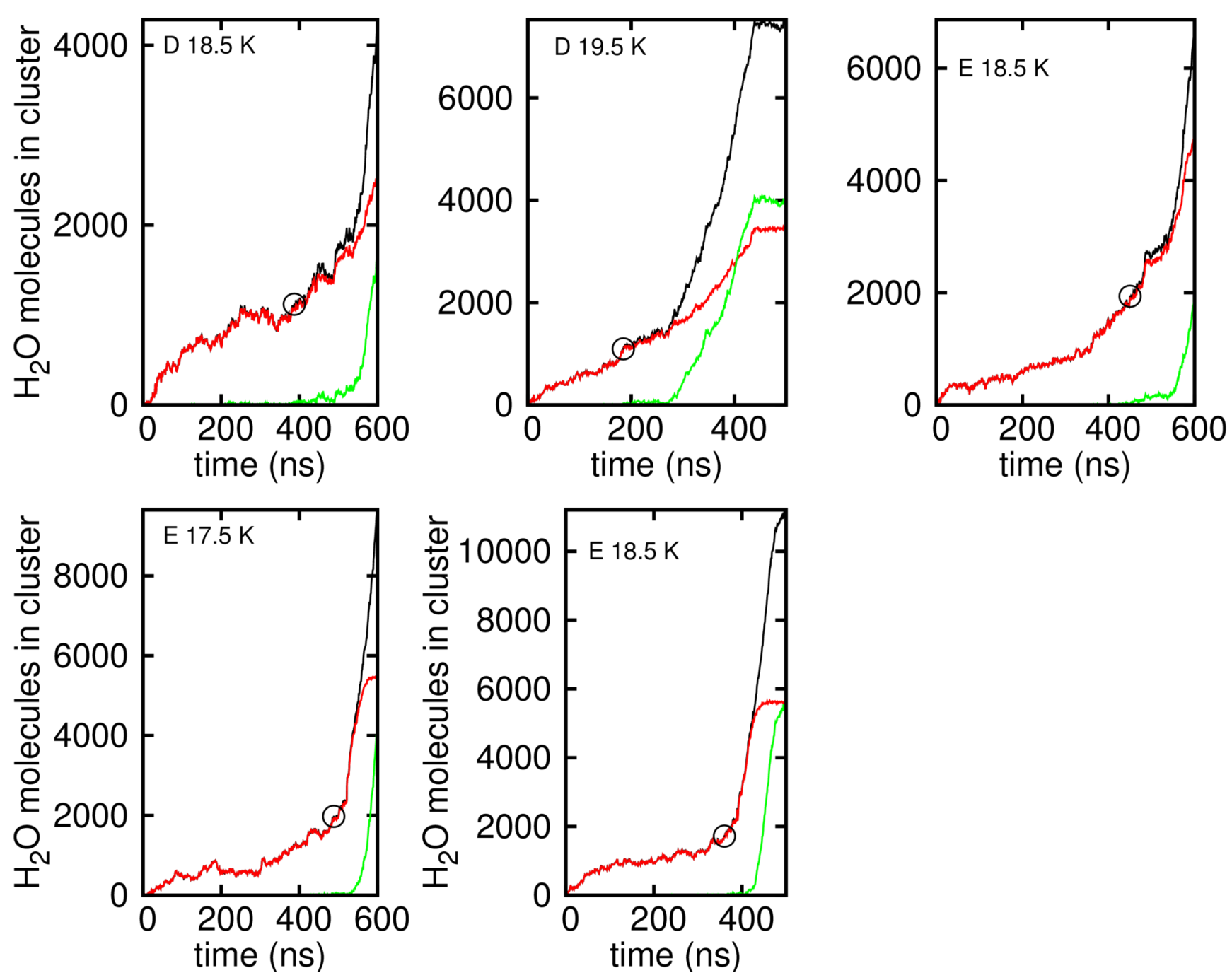

FIG. S4. As in Figure S3, but for the TIP4P/2005 model. 


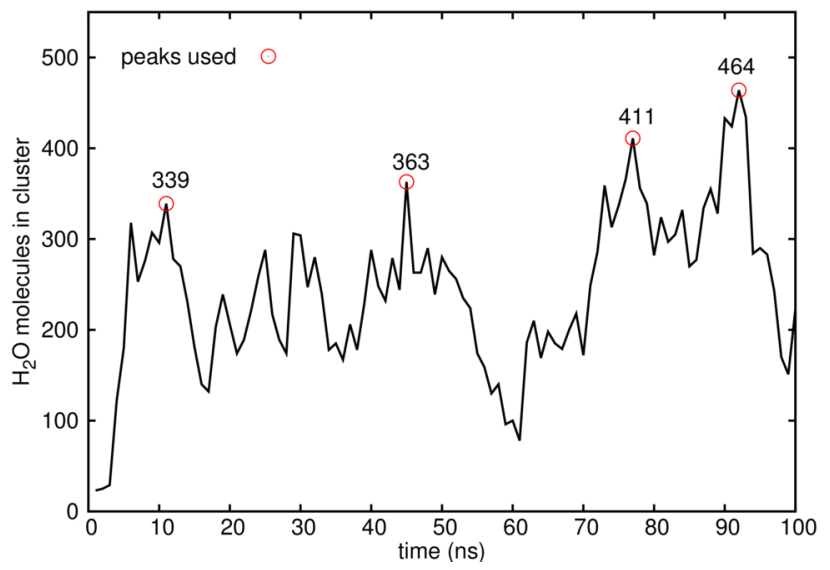

FIG. S5. An example of the peaks selected for the failed cluster plot (Figure 7). This simulation was for the six-site model with plate $\mathrm{C}$ at $\Delta T_{s}=19 \mathrm{~K}$. 

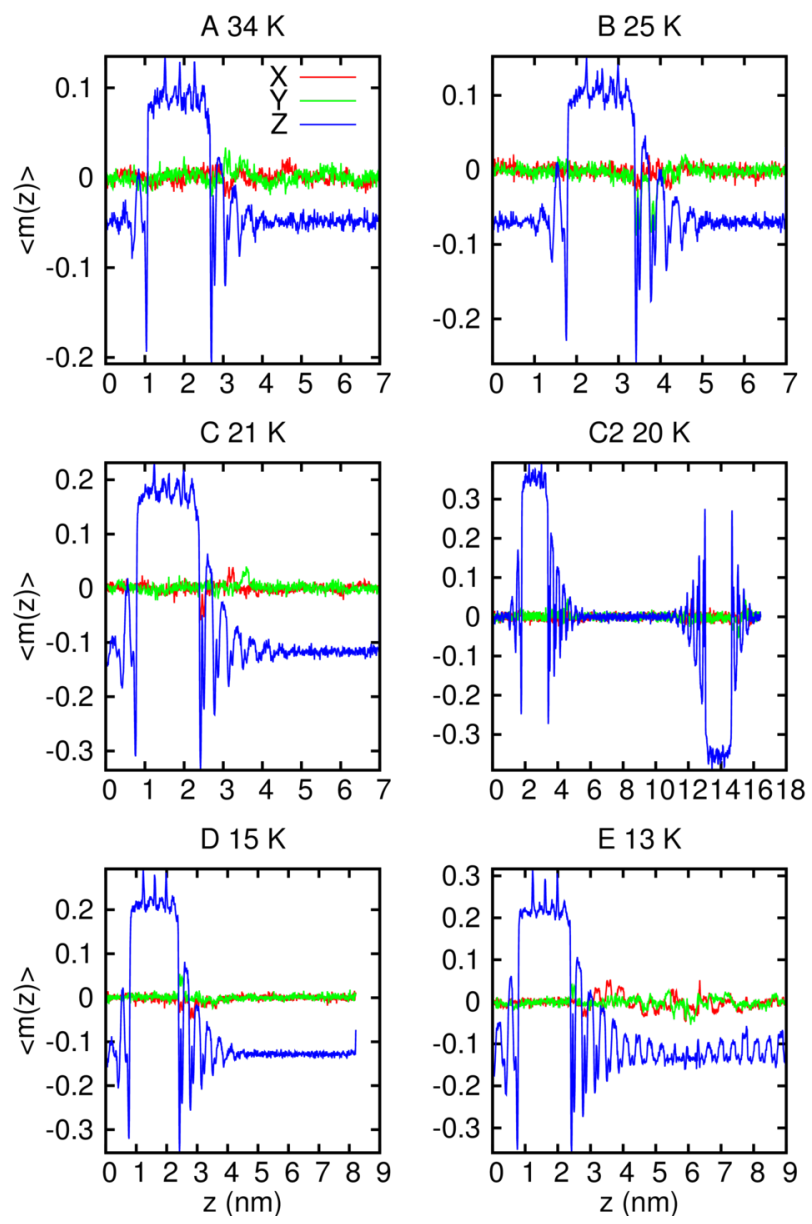

FIG. S6. Polarization profiles of water at $\Delta T_{s}$ values $1 \mathrm{~K}$ above where ice nucleation was observed. Red, green, and blue represent the $X, Y$ and $Z$ components, respectively. The system label and the $\Delta T_{s}$ value are given in the legend. In all systems except $\mathrm{C} 2$, the silver exposed face of the AgI disk is directed outward along the positive $\mathrm{Z}$ axis. For $\mathrm{C} 2$, the silver exposed faces are directed towards each other, such that any long-range fields cancel. 

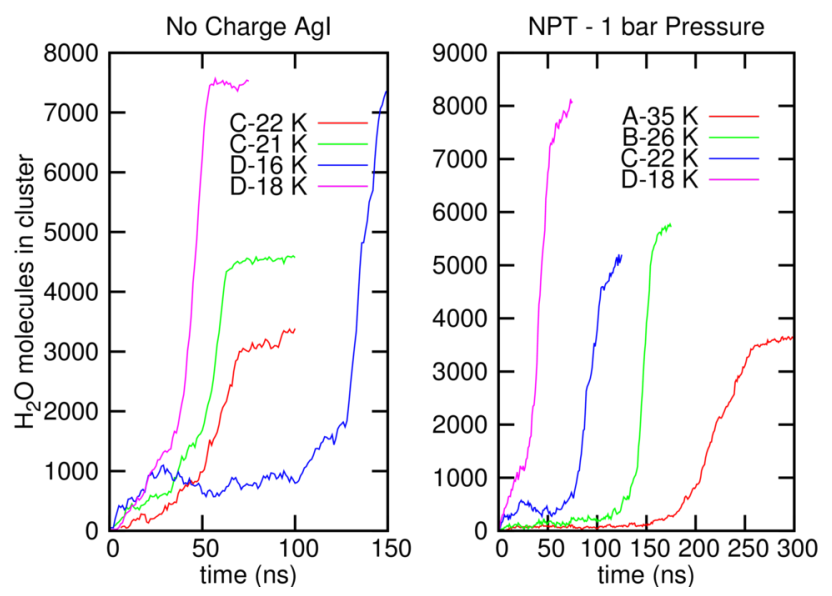

FIG. S7. Ice cluster size vs time for simulations done with neutral Ag and I atoms (left panel) and with pressure coupling maintaining the pressure of the simulation at 1 bar. Keys show the system and $\Delta T_{s}$ for each simulation. 

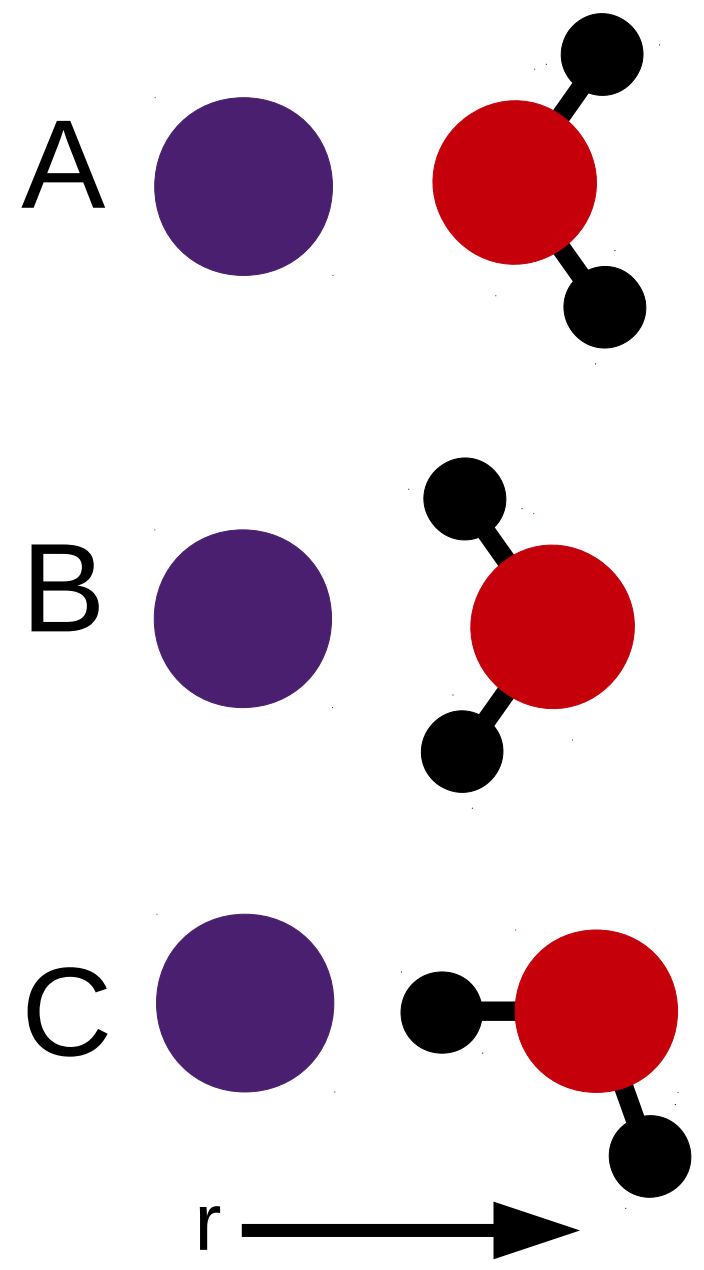

FIG. S8. Orientations of the water molecule used to obtain the pair potentials shown in Fig. S9. The purple circles represent an ion (Ag or I), and the red and black circles represent the oxygen and hydrogen atoms of water, respectively. 

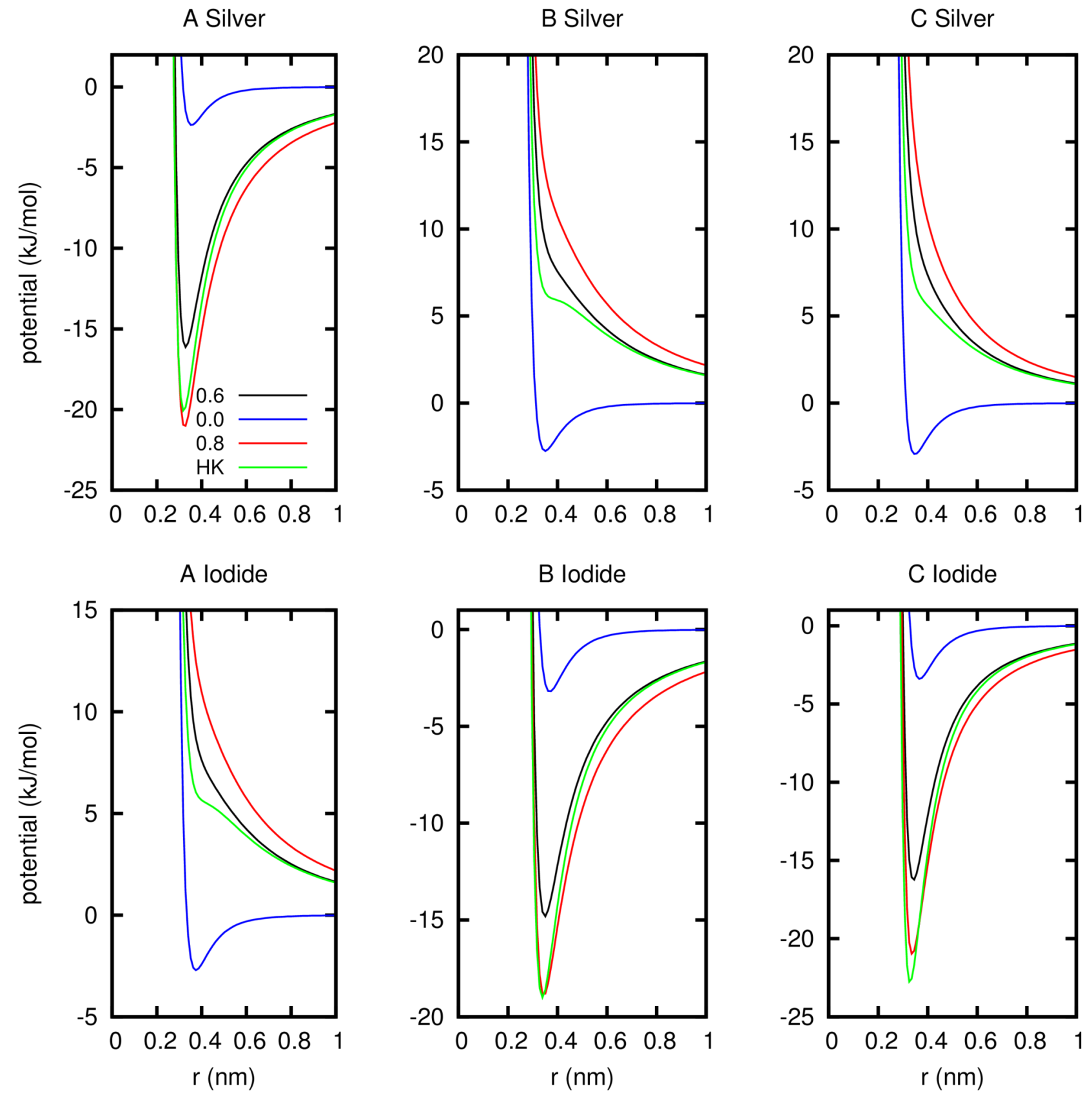

FIG. S9. Comparison of pair potentials for the configurations shown in Fig. S8. The green curve represents the HK potential; the other curves neglect the induction term but vary the charges on the Ag and I ions. The black curve is for charges of $\pm 0.6 \mathrm{e}$ (these are the charges of the HK model), and the red and blue curves are for charges of $\pm 0.8 \mathrm{e}$ and zero, respectively. $r$ is the distance between the ion center and the oxygen atom of the water molecule. In Ref. 2, it is shown that the potentials represented by the black, red and blue curves all result in ice nucleation. 